\title{
IDEALS IN A CERTAIN BANACH ALGEBRA
}

\section{ALVIN HAUSNER}

1. Introduction. The purpose of this paper is to improve a result of Bertram Yood in his paper [2]. We are concerned with a complex commutative Banach algebra $X$ and a compact Hausdorff space $\Omega$. Let $C(\Omega, X)$ denote the set of all continuous functions defined over $\Omega$ with values in $X . C(\Omega, X)$ is a complex commutative $B$-algebra if we define addition, multiplication, and scalar multiplication in the natural "pointwise" manner and if $|f|_{C(\Omega, X)}=\sup _{a \in \Omega}|f(a)|_{x}$ for $f \in C(\Omega, X)$. Here, ||$x$ denotes the norm in $X$. Yood proves, in Lemma 5.1 of his paper, that if $X$ has a unit and if every maximal ideal in $C(\Omega, X)$ is of the form $\left\{f \in C(\Omega, X) \mid f\left(a_{0}\right) \in M_{0}\right\}$ with $a_{0} \in \Omega$ and $M_{0}$ a maximal ideal in $X$, then $\operatorname{Tr}(C(\Omega, X))$, the space of maximal ideals in $C(\Omega, X)$ topologized in the Gelfand sense, is homeomorphic with $\Omega \times \mathfrak{T}(X)$, i.e., the topological product of $\Omega$ with the space of maximal ideals in $X$. We will show that all the maximal ideals in $C(\Omega, X)$ are necessarily of the above form and, further, Yood's result on $\mathfrak{N}(C(\Omega, X))$ is true even if $X$ lacks a unit. If $X$ lacks a unit element, then $C(\Omega, X)$ lacks a unit and $\mathscr{T}(X), \operatorname{TC}(C(\Omega, X))$ denote the spaces of all regular maximal ideals in $X$ and $C(\Omega, X)$ respectively.

2. Proof of theorem. Before establishing two lemmas which we will employ in proving our theorem, we point out that the $B$-algebra $X$ can be considered as contained as a subset of $C(\Omega, X)$. This is accomplished merely by identifying each $x \in X$ with the constant mapping $\Omega \rightarrow x$. The elements of $X$ will thus be viewed as constants in $C(\Omega, X)$.

Lemma 1. Let $f \in C(\Omega, X)$ and $\epsilon>0$. Then there exist $x_{i} \in X$ and continuous complex-valued functions $f_{i}$ defined over $\Omega(i=1,2, \cdots, n)$ such that $\left|f-\sum_{i=1}^{n} x_{i} f_{i}\right|_{C(\Omega, X)} \leqq \epsilon^{1}$

Proof. Let $S=f(\Omega)$. $S$ is a compact set in $X$ since $\Omega$ is compact and $f$ is continuous. Take spheres of radius $\epsilon$ about each point in $S$. This is an open covering of $S$ and, since $S$ is compact, there is a finite open

Received by the editors April 7, 1956 and, in revised form, May 29, 1956.

1 As the proof will show, this lemma is true if $X$ is only a Banach space. The author is grateful to Dr. G. W. Booth for supplying the proof of the lemma and for pointing out that the method of a partition of unity subordinate to a covering of a compact set in a $B$-space is contained in the New York University lecture notes of L. Bers Introduction to topology. 
subcovering $S\left(x_{i}, \epsilon\right)$ of $S(i=1,2, \cdots, n), x_{i} \in S$. We may find a partition of unity subordinate to this covering, i.e., we may find continuous functions on $S, \lambda_{i}(x)(i=1,2, \cdots, n)$, such that $0 \leqq \lambda_{i}(x) \leqq 1$, $\lambda_{i}(x)=0$ on $S-S\left(x_{i}, \epsilon\right)$ and $\sum_{i=i}^{n} \lambda_{i}(x) \equiv 1$ for $x \in S$. To see this, define $\mu_{i}(x)=\operatorname{dist}\left(x, S-S\left(x_{i}, \epsilon\right)\right)$ for $i=1,2, \cdots, n$ where dist $\left(x, S-S\left(x_{i}, \epsilon\right)\right)$ denotes the distance from $x \in S$ to the set $S-S\left(x_{i}, \epsilon\right)$. Each $\mu_{i}(x)$ is continuous and $\geqq 0$. We see that $\mu_{i}(x)=0$ if $x \in S-S\left(x_{i}, \epsilon\right), \mu_{i}(x)>0$ if $x \in S\left(x_{i}, \epsilon\right)$. Write $\mu(x)=\sum_{i=1}^{n} \mu_{i}(x)$. Clearly $\mu(x) \neq 0$ for all $x \in S$ since $\left\{S\left(x_{i}, \epsilon\right)\right\}$ is a covering of $S$. Define $\lambda_{i}(x)=\mu_{i}(x) / \mu(x)$ for $x \in S$. These functions make up the desired partition of unity.

Let $\mathfrak{e}\left(x_{1}, x_{2}, \cdots, x_{n}\right)$ denote the convex hull of the $x_{i}$ 's, i.e., $y \in \mathcal{C}\left(x_{1}, x_{2}, \cdots, x_{n}\right)$ if and only if there exist numbers $r_{1}, r_{2}, \cdots, r_{n}$ $\geqq 0$ such that $\sum_{i=1}^{n} r_{i}=1$ and $y=\sum_{i=1}^{n} r_{i} x_{i}$. Using the $\lambda_{i}(x)$ we may define $T: S \rightarrow \mathfrak{e}\left(x_{1}, x_{2}, \cdots, x_{n}\right)$ as follows: $T x=\sum_{i=1}^{n} \lambda_{i}(x) x_{i}(x \in S)$. $T$ is continuous and, further, $|T x-x| \leqq \epsilon$ for all $x \in S$. For, $|T x-x|$ $=\left|\sum_{i=1}^{n} \lambda_{i}(x) x_{i}-x\right|=\left|\sum_{i=1}^{n} \lambda_{i}(x) x_{i}-\left(\sum_{i=1}^{n} \lambda_{i}(x)\right) x\right|=\mid \sum_{i=1}^{n} \lambda_{i}(x)$ $\cdot\left(x_{i}-x\right)\left|\leqq \sum_{i=1}^{n} \lambda_{i}(x)\right| x_{i}-x \mid \leqq \epsilon$. To complete the proof, define $f_{i}=\lambda_{i}(f)$. Then, $|T f(a)-f(a)|=\left|\sum_{i=1}^{n} x_{i} \lambda_{i}(f(a))-f(a)\right| \leqq \epsilon$ for all $a \in \Omega$. q.e.d.

LEMMa 2. Every, not identically zero, continuous multiplicative linear functional in the B-algebra $C(\Omega, X)$ is of the form $\phi f=\phi_{M} f(a)$ for some $a \in \Omega, M \in \mathfrak{M}(X)$. Here, $\phi_{M}$ denotes the Gelfand homomorphism from $X$ onto the complex numbers associated with $M$.

Proof. Suppose, firstly, that $X$ has a unit $e$ and that $|e|=1$ (if $|e|$ were not 1 we could renorm $X$ so as to achieve this). Then $\phi$ is a nonzero continuous multiplicative linear functional on $e C(\Omega)$ $=\{e f \in C(\Omega, X) \mid f \in C(\Omega)\}$ (here, $C(\Omega)$ denotes the $B$-algebra of continuous complex-valued functions defined over $\Omega$ ). Also $\phi$ is a nonzero multiplicative linear functional on $X \subset C(\Omega, X)$. The last two statements concerning $\phi$ can be proved as follows: If $\phi$ were identically zero on $e C(\Omega)$ or $X$, then $\phi(x f)=\phi(e f) \cdot \phi(x)=0$ for all $f \in C(\Omega)$ and all $x \in X$. Since linear combinations of functions $x f$ with $f \in C(\Omega), x \in X$ are dense in $C(\Omega, X)$ by Lemma 1 , this would mean $\phi$ is identically zero in $C(\Omega, X)$ contrary to assumption.

It is easy to see that $e C(\Omega)$ is isometrically isomorphic with $C(\Omega)$. Hence, as is well known, there exists an $a \in \Omega$ such that $\phi(e f)=f(a)$ with $f \in C(\Omega)$. Also there exists an $M \in \mathscr{T}(X)$ such that $\phi(x)=\phi_{M}(x)$ for all $x \in X \subset C(\Omega, X)$.

Suppose, now, that $f$ is an arbitrary function in $C(\Omega, X)$. By Lemma 1 , there exists a sequence $f_{n} \in C(\Omega, X)$ such that $f_{n} \rightarrow f$ in $C(\Omega, X)$-norm 
and furthermore $\phi\left(f_{n}\right)=\phi_{M} f_{n}(a)$. Since $\phi$ is continuous we have $\phi f=\phi_{M} f(a)$.

Suppose that $X$ lacks an $e$. Then we imbed $X$, isometrically and isomorphically, in a Banach algebra $X^{\prime}$ with unit $e$ in such a way that the maximal ideals of $X^{\prime}$ are the regular maximal ideals of $X$ and $X$ itself. This gives rise to an additional homomorphism of $X^{\prime}$ onto the complex numbers, namely $\phi_{X}$, where $\phi_{X}(x)=0$ if $x \in X$ and $\phi_{X}(\lambda e)=\lambda$ for all complex numbers $\lambda$. The space $\mathfrak{T}\left(X^{\prime}\right)$ is the one-point-compactification of $\mathfrak{T}(X)$ by $\phi_{X}$. By what we have already proved, the nonzero multiplicative functionals in $C\left(\Omega, X^{\prime}\right)$ are of the form $\phi_{M} f(a)$ and the additional functionals $\phi_{X} f(a)$. These latter functionals are all identically zero on $C(\Omega, X)$ so that the most general nonzero multiplicative functionals on $C(\Omega, X)$ are of the form $\phi_{M} f(a)$ with $M \in \Re(X), a \in \Omega$. q.e.d.

Corollary. The only regular maximal ideals in $C(\Omega, X)$ are of the form $\{f \in C(\Omega, X) \mid f(a) \in M\}$ with $a \in \Omega, M \in \mathscr{T}(X)$.

THEOREM. $\mathfrak{T C}(C(\Omega, X))$ is homeomorphic with $\Omega \times \mathfrak{T}(X)$.

Proof. By the corollary, above, there is a 1-1 correspondence between the points of $\mathfrak{T}(C(\Omega, X))$ and those of $\Omega \times \mathfrak{T}(X)$. Now, the topology in $\mathscr{T}(C(\Omega, X))$ is that induced by the family $\mathcal{I}$ which consists of the functions $g_{f}(f \in C(\Omega, X))$ defined on $\Omega \times \mathfrak{T}(X)$ by $g_{f}(a, M)$ $=\phi_{M} f(a)$. We must show that this topology is identical with the product topology. For this purpose we use the following result (see $[1$, p. 12]):

If $\mathcal{F}$ is a family of continuous complex-valued functions vanishing at infinity on a locally compact space $\Pi$, separating the points of $\Pi$ and not all vanishing at any point of $\Pi$, then the topology induced on $\Pi$ by $\mathcal{F}$ is identical with the given topology of $\Pi$.

We take $\Pi=\Omega \times \mathfrak{T}(X)$ and define $\mathcal{F}$ as follows. For each positive integer $n$ and each choice of $f_{1}, \cdots, f_{n} \in C(\Omega), x_{1}, \cdots, x_{n} \in X$, there is a function $h$ defined on $\Omega \times \mathfrak{T}(X)$ by $h(a, M)=\sum_{i=1}^{n} \phi_{M}\left(x_{i} f_{i}(a)\right)$. Let $\mathcal{F}$ be the family of all functions $h$ so defined. $\Pi$ is locally compact since $\Omega$ is compact and $\mathscr{T}(X)$ is locally compact. Further, every function in $\mathcal{F}$ is continuous in $(a, M)$ over $\Omega \times \mathfrak{T}(X)$ since this is true for the functions $\phi_{M} f(a)$ with $f \in C(\Omega, X)$ (see [2, Lemma 2.2]).

Not all functions in $\mathcal{F}$ vanish at any point $\left(a_{0}, M_{0}\right) \in \Omega \times \mathfrak{N}(X)$. For, let $x \in X$ be such that $x \in M_{0}$ and pick $f \in C(\Omega)$ such that $f\left(a_{0}\right) \neq 0$. Then $\phi_{M_{0}}\left(x f\left(a_{0}\right)\right) \neq 0$.

The functions in $\mathcal{F}$ separate points in $\Omega \times \mathfrak{T}(X)$. Suppose $(a, M)$ $\neq(b, N)$ with $a \neq b$. If $M=N$, take $x \notin M$ and $f \in C(\Omega)$ such that 
$f(a) \neq f(b)$. Then $\phi_{M}(x f(a)) \neq \phi_{N}(x f(b))$. If $M \neq N$, find an $x \in M, x \notin N$ and $f \in C(\Omega)$ such that $f(a)=0$ and $f(b) \neq 0$. Then $\phi_{M}(x f(a)) \neq \phi_{N}(x f(b))$. If $a=b$ so that $M \neq N$ we may find $x \in M, x \notin N$. Choose $f \in C(\Omega)$ such that $f(a)=f(b) \neq 0$; then $\phi_{M}(x f(a)) \neq \phi_{N}(x f(b))$.

Finally we show that functions in $\mathcal{F}$ vanish at $\infty$ in $\Omega \times \mathfrak{N}(X)$. Suppose $\epsilon>0$ is given. If $\sum_{i=1}^{n} \phi_{M}\left(x_{i} f_{i}(a)\right) \in \mathcal{F}$, then $\left|\sum_{i=1}^{n} f_{i}(a) \phi_{M}\left(x_{i}\right)\right|$ $\leqq \epsilon$ if $(a, M) \notin \Omega \times\left(\cup_{i=1}^{n} \mathfrak{e}_{i}\right)$ where $\left|\phi_{M}\left(x_{i}\right)\right|<\delta$ if $M \notin \mathfrak{C}_{i}, \mathfrak{e}_{i}$ being compact sets in $\operatorname{Tr}(X)(i=1,2, \cdots, n)$, and where $\delta<\epsilon / n K$ with $K=\sup _{1 \leq i \leq n} \sup _{a \in \Omega}\left|f_{i}(a)\right|$. The sets $\mathcal{C}_{i}$ exist because each $\phi_{M}\left(x_{i}\right)$ vanishes at infinity in $\mathfrak{N}(X)$. Further, $\Omega \times\left(\bigcup_{i=1}^{n} \mathfrak{C}_{i}\right)$ is compact in $\Omega \times \mathfrak{M}(X)$ so that each function in $\mathcal{F}$ vanishes at $\infty$ in $\Omega \times \mathfrak{M}(X)$.

Using the result quoted in the beginning of the proof we have shown that the product topology of $\Omega \times \mathscr{M}(X)$ is identical with that induced by our family $\mathcal{F}$. Since $\mathcal{F}$ is smaller than the family $\mathcal{I}$ we see that the topology induced by $\mathcal{J}$ on $\Omega \times \mathscr{T}(X)$ is stronger or equal to the topology induced by $\mathcal{F}$. Since the topology of $\mathfrak{T}(C(\Omega, X))$ is precisely that induced on $\Omega \times \mathfrak{M}(X)$ by the family $\mathcal{J}$, the proof will be completed by showing that the $\mathcal{F}$ - and $\mathcal{g}$-topologies on $\Omega \times \mathfrak{M}(X)$ are identical. The family $\mathcal{F}$ is contained in and is dense in $\mathcal{J}$ in the uniform norm for continuous functions. For, suppose $f \in C(\Omega, X)$. Then, by Lemma 1 , we can find $\sum_{i=1}^{n} x_{i} f_{i}, f_{i} \in C(\Omega), x_{i} \in X$ such that $\left|f-\sum_{i=1}^{n} x_{i} f_{i}\right|_{C(\Omega, X)} \leqq \epsilon$. This means sup $\left|\phi_{M} f(a)-\sum_{i=1}^{n} \phi_{M}\left(x_{i} f_{i}(a)\right)\right|$ $\leqq \epsilon$, where the sup is taken over all $(a, M) \in \Omega \times \mathfrak{N}(X)$. Hence $\mathcal{F}$ is dense in $\mathcal{J}$ in the uniform norm and it follows that the $\mathcal{F}$ - and $\mathcal{J}$-topologies are identical. q.e.d.

3. Concluding remarks. The essential ideas in this paper are direct outgrowths of the author's Yale doctoral thesis Group algebras of vector-valued functions. Just as in the thesis we can show, using the characterization of $\mathfrak{M}(C(\Omega, X))$ as $\Omega \times \mathfrak{M}(X)$, that $C(\Omega, X)$ is regular if and only if $X$ is regular, and that every proper closed ideal in $C(\Omega, X)$ is contained in a regular maximal ideal if $X$ satisfies certain additional conditions. Further, results on kernels and hulls could be obtained in $C(\Omega, X)$. All these can be proved by minor and obvious changes in the proofs of the thesis.

\section{REFERENCES}

1. L. H. Loomis, An introduction to abstract harmonic analysis, New York, 1953.

2. B. Yood, Banach algebras of continuous functions, Amer. J. Math. vol. 73 (1951) pp. $30-42$.

Brooklyn College 